\title{
LA EXISTENCIA Y EL ARGUMENTO ONTOLÓGICO
}

En este trabajo se discute la tesis que niega a la existencia la calidad de predicado lógico. Ya Aristóteles había dicho que "la existencia no puede pertenecer nunca a la esencia de una cosa" (Analit. Post. c. 7), pero no fue sino hasta los primeros intentos de refutar el argumento ontológico, que la tesis fue presentada más explícitamente. De ahí en adelante, la tesis se ha ido formulando cada vez con más precisión, claridad y fuerza explicativa. Estamos lejos, sin embargo, de haber llegado a una situación plenamente satisfactoria. La bibliografía actual presenta importantes discrepancias en cuanto a la formulación de la tesis. No se ha llegado a un acuerdo sobre qué formulaciones sean inaceptables, ni sobre cuáles sean menos engañosas. El caso de las tautología referenciales es aún peor: según la versión de Rusell, no tienen sentido; Pears, en cambio, no sólo sostiene que tienen sentido, sino que formula la tesis diciendo precisamente que la existencia produce tautologías referenciales. Strawson, además, presenta un contexto en el que a su entender la existencia si cumple el papel de predicado lógico.

Intentaré aproximarme a una formulación que dé razón de los siguientes hechos lingüísticos: i) el argumento ontológico no es válido; ii) las proposiciones existenciales son, en general, contingentes; iii) los adjetivos cuantificadores no tienen siempre sentido cuando 'existe(n)' ocupa el lugar de otros predicados gramaticales; $i v)$ las oraciones como "esto existe", "este tigre manso existe", o "esta mancha roja existe" no pueden ser falsas (tautologias referenciales).

\section{El argumento ontológico}

El argumento ontológico presenta la siguiente estructura:

a) se redefine la palabra 'Dios', de modo que sea el ser que presente una característica que llamaré ' $D$ '. Ésta puede significar "el ser mayor que concebirse pueda", "el ser a cuya comparación ningún otro es mejor", "el ser perfecto". Ast, 'Dios' es igual por definición a ' $(\eta x)(D x)$ '.

b) se muestra que la expresión 'Dios' no puede referirse a más de un objeto: no más de un objeto puede tener la característica $D$.

$c$ ) se alega que el concepto ' $D$ ' implica existencia. Si por ' $E$ ' entendemos 'existe', esto puede expresarse así: " $(x)(D x \supset E x)$ " es una verdad analitica. Esto, en virtud de que un ser no existente no puede ser el mayor, el más perfecto: el que existiera sería mayor, más perfecto. 
d) se deduce que "Dios no existe" es una contradicción pues pretende referirse a un ser que por definición tiene que existir, y pasa inmediatamente a negar su existencia.

e) se concluye que "Dios existe" es una verdad necesaria y que, por tanto, Dios existe por necesidad lógica.

Ya Santo Tomás indicó que la definición en a) no puede considerarse un análisis correcto de la palabra 'Dios', según el uso común de la voz. Pero no viene a cuento discutir aquí las relaciones lógicas entre el concepto asf definido y las proposiciones en que aparece el concepto ordinario de Dios. Lo que nos interesa es refutar que la existencia de $(\eta x)(D x)$ sea necesaria. Asf tomemos la definición en a) como estipulativa, el problema no deja de ser vigente. En cuanto a $b$ ), no vamos tampoco a discutirlo: asumiremos que parte del significado de ' $D$ ' es que $(x)(y)(D x \supset x=y)$. Para discutir la verdad de $c$ ), es preciso explicar primero bajo qué condiciones un enunciado de la forma " $(x)(F x \supset G x)$ " es una verdad de la lógica. Este enunciado, que es equivalente a " $F \subset G$ ", nos dice que de todo objeto del que podamos decir con verdad que es $F$, podremos decir que es $G$, o sea que la clase de los objetos $F$ es una subclase de la clase de los objetos $G$. Ahora bien, si una verdad semejante va a ser analftica, si va a ser una verdad necesaria, deberá seguirse del significado de ' $F$ ' y de ' $G$ '. $Y$ esto no puede ser sino de la siguiente forma: cuando digo ' $F a$ ' (o ' $a \in F$ ), afirmo que el objeto a cúmple las condiciones necesarias y suficientes que, según el significado de ' $F$ ', un objeto debe tener para que pueda afirmarse con verdad que es un $F$ (afirmo que el objeto a cumple las condiciones necesarias y suficientes para pertenecer a la clase $F$ según la definición de esta clase). De modo que decir que una proposición como " $(x)(F x \supset G x)$ " es una verdad lógica es decir que condición necesaria para que un objeto sea un $F$, según el significado de ' $F$, que cumpla todas las condiciones necesarias y suficientes para ser un $G$, ségún el significado de ' $G$ '. Vamos a decir que un predicado implica a otro cuando esto sucede. Decir, pues, que ' $F$ ' implica a ' $G$ ', es decir que no estamos dispuestos, según las convenciones de nuestro lenguaje, a sostener que un objeto es $F$ si no es, primero, $G$. Lo que se ha hecho en a) no es otra cosa que establecer ciertas convenciones lingüisticas relativas a la palabra 'Dios'. Convenciones que regulan los usos de la palabra 'Dios', que nos dicen cuáles son las condiciones necesarias para que la aplicación de la palabara dé por resultado un enunciado verdadero. En $b$ ) y en $c$ ) se han hecho explicitas. Se ha puesto como condición necesaria de la verdad de " $a$ es Dios" que a exista. Se ha mostrado que si es verdadera una oración como "a es Dios", tämbién será verdadero que $a$ existe.

No hay ninguna razón que nos impida inventar los conceptos que más nos acomoden. La forma en que se hace esto es la siguiente: se toma un signo, una palabra, y se establecen condiciones necesarias y suficientes para su apli- 
cación verdadera. Estas condiciones son parte de lo que llamamos, en general, reglas de uso de un término. Pues bien: no tenemos ninguna limitación a esta actividad de impartir reglas a un término. Es absoluta nuestra libertad en este sentido. Pero dar determinadas reglas a un término es darle cierto significado. A eso nos comprometemos cuando establecemos las reglas. El imperativo lógico que nos prohibe dar reglas contrarias o contradictorias no es ninguna limitación: podemos inventar las reglas que queramos, pero dar reglas que se anulen las unas a las otras, como dar reglas que no entendamos, no es, en verdad, sino fracasar en el intento de dar alguna regla.

De dos modos pueden dársele a un término reglas que limiten los casos de su aplicación verdadera. En ambos mediante la definición del término. En el primero, se establece directamente la condición. Por ejemplo, cuando entre las condiciones de aplicación verdadera de 'perro' queremos incluir el requisito de que sea un ente orgánico, podemos establecer directamente ese requisito. Pero también podemos hacerlo - y éste es el segundo modo- exigiendo que el objeto sea un animal. Esta segunda forma consiste pues, en incluir el requisito a través de otro requisito que lo incluya. Si se hace de esta segunda forma, para asegurarse de que hemos incluido el requisito que queremos, tenemos que demostrar que está incluido en el sentido del término intermediario mediante el cual lo queremos introducir (en este caso 'animal').

En el caso del argumento ontológico se ha elegido el segundo camino: para incluir la existencia entre los requisitos para que algo sea Dios, se ha recurrido a un término intermediario tal como 'ser más perfecto'. Es claro que se pudo recurrir al primer camino y establecer directamente que para que algo sea Dios tiene que existir. Ast', ' $D x$ ' puede definirse como sigue ' $D x$ ' $=d f$ $(C x \cdot E x)$, donde ' $C x$ ' se refiere al conjunto de las demás propiedades de Dios y 'Ex' establece el requisito de existencia para la aplicación verdadera del concepto $D x$. Si esto es así, en vano discutiremos si el concepto 'ser perfecto' o algunos de los mencionados en a) implique o no la existencia; inútil será. ingeniarnos para inventar algún otro atributo de Dios que implique existencia. Si queremos, podemos incluirla de plano; estos rodeos son innecesarios, 'su utilidad es sólo la de disfrazar el problema. En este engaño caen, por ejemplo, quienes critican al argumento de petitio principii, como si el truco consistiera en haber deslizado la conclusión dentro de la premisa. Podemos decir que el concepto 'Dios' implica existencia, sin que por ello la existencia de Dios sea lógicamente necesaria. Vamos a tratar de encontrar pues, la falacia en $d$ ), y no en las premisas anteriores, pero primero demos a $d$ ) la plausibilidad que merece, como la merecen todas las falacias.

Concedamos, pues, que una de las condiciones necesarias para que " $x$ es Dios" sea verdadera es que el objeto $x$ exista. Si esto es asi, de la verdad de " $a$ es Dios" se podrá concluir " $a$ existe" igual que se puede concluir " $a$ es un ser perfecto". Hemos aceptado también que parte del significado de 'Dios' 
es que no puede aplicarse a ningún otro objeto. Pero si esto es asi, entonces la expresión 'Dios' puede servir para identificar al objeto que reúna las condiciones necesarias y suficientes para ser llamado 'Dios'. Es claro que este modo de identificar estará condicionado a que haya un objeto que reúna las condiciones. De esta manera pueden formarse oraciones como "Dios es vengativo" en las que la palabra 'Dios' sirve para indicarnos de quién decimos que es vengativo. Pero, puesto que la existencia es condición necesaria de la aplicación de 'Dios', y puesto que decimos que nos referimos al objeto que reúna todas las condiciones necesarias que hemos aparejado a la palabra Dios, hemos de concluir que todas las proposiciones de la forma "Dios...", es decir, las que tengan 'Dios' como sujeto, todas implican que el objeto del que se habla existe, pues que exista es una de esas condiciones. Ahora bien: "Dios no existe" es de la forma "Dios...", luego implica, como todas las otras "Dios existe". Pero siempre que $\sim p$ implique lógicamente a $p$, lo que sucede es que $p$ es una verdad de la lógica. Así queda demostrado que "Dios existe" es una verdad de la lógica. Y si por alguna razón oculta no gustamos aquí la paradoja, no es necesario recurrir a la isla perfecta llena de riquezas que nos ofrece Gaunilón con la misma necesidad lógica de Dios, ni tenemos tampoco que atenernos al caballo alado de Gassendi: como hemos dicho, podemos construir todas las descripciones determinadas que podamos imaginar, incluyendo en ellas la existencia en forma explícita. Sin quebrarnos la cabeza para deducir la existencia de otro predicado podemos intentar los menos ingeniosos pero igualmente paradójicos enunciados de la forma "El existente Emperador de Oaxaca no existe".

No es difícil mostrar que el argumento se rompe cuando negamos que, en la oración "Dios existe", Dios es el sujeto lógico y la existencia, por tanto, no se predica de Dios. Esto querria decir que 'Dios', en esa oración, no cumple el papel de identificar al objeto que cumple las condiciones necesarias y suficientes para que se diga que ese objeto es Dios. Si decimos que la oración no debe entenderse como una oración de la forma "Dios...", lo que estamos diciendo es que no estamos identificando al $(\eta x)(D x)$, sino a otro objeto; que alli 'Dios' cumple otro papel. Esto nos ayudará a ver qué es lo que queremos negar, precisamente, cuando negamos que la existencia sea un predicado lógico. Negamos que la oración esté sujeta a cierto tipo de condiciones para ser un enunciado. Tanto Russell como Strawson admitirian que si Dios fuera el sujeto lógico de "Dios existe", entonces, para que la oración pudiera ser un, enunciado sería necesario que hubiera un objeto que satisfaciera al concepto 'Dios'. (Que hubiese un objeto del que fuera verdadero decir que es Dios.) Decir que 'existe' no se predica de Dios es decir que el enunciado se logra sin que un objeto responda a todas las condiciones necesarias y suficientes para ser Dios. 


\section{El problema de la predicación}

Tal como he planteado el argumento ontológico, no es difícil que se confunda con el famoso problema de la predicación. Por esta razón, y porque este problema nos ayudará a caracterizar la estructura sujeto-predicado, voy a explicarlo.

i) Sea ' $S$ ' la oración "El Emperador de Oaxaca no existe".

ii) $S$ es no sólo perfectamente significativa sino que todos sabemos que es verdadera.

iii) $S$ es una oración en la que se predica la no existencia, y se predica precisamente del Emperador de Oaxaca.

iv) Pero siempre que se predique algo del Emperador de Oaxaca, para que lo que se diga sea verdadero será necesario que el Emperador cumpla con las condiciones que ese predicado implica; pero para que cumpla o deje de cumplir esas condiciones es necesario que el Emperador exista.

v) De manera que una precondiçión para que se cumplan las condiciones de verdad de una predicación es que el sujeto lógico exista.

vi) $S$ no puede, pues, ser verdadera, porque una precondición para que pueda ser verdadera (o falsa) es precisamente lo que pide el predicado que no suceda para aplicarse con verdad.

vii) Pero, puesto que este argumento vale para cualquier oración de la forma ' $x$ no existe', el problem ade la predicación es el de explicar que no todas las oraciones de esa forma son falsas, pues sabemos que muchas no lo son. De lo que se trata es de dar a la expresión 'no existe' una interpretación que nos permita decir lo que comúnmente decimos con ellas, que es contingente.

Un argumento semejante puede llevarnos a la conclusión de que ' $x$ existe' tiene que ser verdadera si 'existe' se predica de $x$, puesto que una precondición para que un predicado sea verdadero o falso de un objeto es que el objeto exista, ya que no tiene sentido averiguar si un objeto cumple las condiciones que impone un predicado si no hay objeto.

Todas las soluciones que han podido darse a este problema coinciden en negar que la existencia de Dios sea una precondición para que "Dios existe" tenga valor de verdad. Niegan que la predicación de existencia tenga. esa precondición; todas niegan que para poder lograr una predicación de existencia (con verdad o con falsedad), haya de poderse identificar el mismo objeto que trata de identificarse con la misma frase-sujeto cuando el predicado es otro. Cuando decimos "Dios es vengativo", el sujeto lógico no es el mismo que cuando decimos "Dios existe". Ha habido muchas formas de conceptuar esto, unas mejores que otras, desde distintos puntos de vista (con 
relación, por ejemplo, at problema ontológico). Podemos decir que es precondición de valor dé verdad de "Dios es vengativo" que se identifique con la expresión 'Dios' a un objeto existente, y que, en cambio, la oración "Dios existe" tiene como precondición el que haya un objeto subsistente. No me interesa aqui discutir el problema de si debamos admitir estos entes en nuestra ontologfá. Lo único que quiero destacar es que el proceso previo a la verificación que se llama 'identificación' es diferente en el caso de los objetos existentes que en el caso de los subsistentes. La distinción está allí decir que el objeto existente no es el sujeto lógico es simplemente indicar que hay una precondición de identificación que no tiene la oración en cuestión. Cuando se dice que un ente subsistente es el sujeto lógico, se afirma lo anterior, y se agrega cuál es la indole de la identificación requerida. En cuanto a esto último, la forma de decidirlo es la de dar condiciones necesarias y suficientes para que se identifique un objeto subsistente (el sujeto del predicado 'existe(n)') o lo que es lo mismo, para que la expresión-sujeto pueda denotar a un miembro del universo de discurso del predicado en cuestión. Porque de lo que se trata es de integrar el universo de discurso del predicado, tener un criterio para decidir si algo pertenece a él. En este momento no discutiré las condiciones de identificación en ese universo de discurso. Lo único que quiero hacer claro es que no pueden ser las mismas que las del universo de discurso de los objetos existentes. En esto coinciden tanto los que usan la terminologia 'objeto subsistente' cuanto los que llaman a los sujetos de las oraciones existenciales 'conceptos' o 'funciones proposicionales'. Lo que se concluye de aqui es que el universo de discurso de 'existe(n)' no puede ser el de los objetos existentes porque toda afirmación de existencia sería tautológica, y toda negación contradictoria. Si no estamos dispuestos a aceptar esta consecuencia es porque tenemos un predicado 'existe(n)' que sirve para decir verdades empiricas: no puede ser el que tenga ese universo de discurso y sea verdadero de todo y falso de nada.

Para señalar la diferencia entre este argumento y el ontológico, debemos fijarnos en esa rara propiedad que tiene la existencia y que se aduce para decir que no es un predicado: la existencia no añade nada nuevo al concepto. Supongamos que analizamos el uso de una expresión como 'El Emperador de Oaxaca' cuando denota a un sujeto lógico del predicado 'es valiente' en contextos de validez histórica. Puesto que el objeto identificable para que la oración tenga valor de verdad tiene que pertenecer al universo de discurso de los objetos existentes, añadir al sujeto la palabra 'existente', o al predicado 'y existe', no es sino repetir una precondición del valor de verdad de la oración.

Pero el argumento ontológico no parece tratar de añadir inútilmente una precondición que ya tienen estas oraciones (que ya tendrían si 'existe(n)' pudiera ser una condición extra para que la aplicación del predicado dé una 
verdad, no para que tenga valor de verdad). La única forma para que esto fuera así consistiría en admitir que los predicados del lenguaje en que nos movemos tuviesen como universo de discurso a una clase heterogénea compuesta por sujetos lógicos de los que tanto puede predicarse 'existe(n)' como 'no existe(n), objetos que, como ya vimos, son distintos de aquellos que no puetlen identificarse sino como existentes. Decir que son otros objetos no es sino negar que las oraciones existenciales requieran ciertas condiciones de identificación para ser enunciados, para tener valor de verdad. Tanto el argumento ontológico como cierta interpretación de oraciones semejantes a "El existente Emperador de Oaxaca existe" implican ese universo de discurso distinto. Cuando digo que si $x$ no existe $x$ no es Dios, porque si existiera una $y$ que fuese mayor que $x$ y sería entonces Dios, tengo que aceptar que 'Dios' puede ser aplicado a no existentes: aunque todas esas aplicaciones sean falsas, ten. drán que tener sentido. En la terminologia de San Anselmo, el universo de discurso de 'Dios' sería el de objetos que existen en la mente y objetos que existen en la realidad. $O$ sea que no puede ser el de los objetos que existen en la realidad. Es engañoso decir que el universo de discurso es este universo. heterogéneo de objetos existentes y no existentes, porque da la impresión de que este universo incluye el otro, y se trata, en realidad, de universos de distinto orden. Para explicar esto debemos fijarnos primero en una ambiguiedad que presentan todos los predicados de los lenguajes ordinarios. No siempre que se utilicen en actos de predicación, el prerrequisito referencial de identificación del acto ès del mismo tipo. Por ejemplo, cuando decimos que Sancho perdió su rucio, cosa que es verdadera, no es necesario que identifiquemos a Sancho como un personaje histórico, sino que se trata de identificar al Sancho de la novela de Cervantes. Es, pues, el contexto el que nos dirá de un determinado acto de predicación qué requisitos referenciales tiene. Pero aquí hablamos de predicados determinados ya por un contexto. $Y$, hasta ahora, hemos estado omitiendo esto.

La determinación que en el argumento ontológico se da a la palabra 'Dios' vía la determinación que se da a expresiones como 'mayor que', 'perfecto', no tiene sentido sino en un contexto en el que el universo de discurso de las predicaciones normales sea un universo heterogéneo compuesto por entes de los que tanto puede predicarse (tiene sentido hacerlo) que son reales, cuanto que son solamente imaginados. Esto quiere decir que aquí, de la felicidad de una predicación (del hecho de que sea exitosa, tenga valor de verdad) no podrá seguirse que el sujeto es real, a menos que el sujeto incluya esta propiedad en su connotación. Para que una predicación se logre será necesario que un objeto sea identificado en ese universo mixto. De una predicación feliz, por tanto, podrá seguirse que la frase-sujeto puede aplicarse con verdad de un objeto y sólo a uno de ese universo heterogéneo. Esto es lo que se seguirfa del mero hecho de que se haya logrado una predicación en este 
contexto. Pero si, como en el caso de 'Dios', añadimos 'existe' o 'es real' como condición de la aplicación del término, entonces de la predicación feliz de ese objeto se sigue su existencia. Exactamente en la misma forma en que de una predicación exitosa acerca del Emperador de Oaxaca se seguiria que el objeto en cuestión es un Emperador. Pero de aquí no se sigue que 'Dios existe' sea una verdad analítica. Lo que sí sería analítico sería el siguiente condicional: si $x$ es Dios, entonces $x$ existe; si con la palabra 'Dios' identifico algo, entonces Dios existe. Pero puede muy bien ser el caso que en ese universo heterogéneo no se encuentre ningún objeto del que sea verdadero decir que es Dios. $Y$ en esta última oración nos encontramos un segundo sentido de la palabra 'existe(n)'. En el primero, decir ' $x$ existe' es predicar la existencia de uno de los miembros de ese universo heterogéneo. Así, decimos que Pegaso no existe, o que Moctezuma sí existió, y para que estas predicaciones de existencia tengan sentido, es necesario que haya un objeto dentro del universo de discurso que como Pegaso o Moctezuma sea identificado.

Hay, pues, por lo menos dos sentidos de 'existe(n)': a) en el primero se dice que un objeto es real; $b$ ) en el segundo se dice que es real o imaginario; y de esta forma pueden generarse muchos más que la ambigüedad natural de la palabra permite. La distinción entre estos sentidos es impor. tante, pues está relacionada directamente con la tesis que queremos aclarar. Cuando decimos que la existencia no es un predicado, afirmamos que las oraciones existenciales no tienen el mismo tipo de sujeto lógico que las demás, en el contexto en el que nos encontremos. Supongamos, primero, que hablamos de objetos reales, que ese es nuestro universo de discurso. En tal caso, para que nuestras predicaciones se logren, para que produzcan un verdadero enunciado, es necesario que la frase-sujeto (en algunos casos acompañada de un señalamiento) logre identificar algo, dentro de ese universo. Ahora bien: cuando la frase-sujeto tiene connotación, expresaremos, como parte de la condición de identificación, que es necesario que haya un objeto que tenga las propiedades a que se refiere la connotación, que exista el objeto referido. Cuando la frase-sujeto no connota nada, si esto es posible, podremos de todas formas afirmar que se ha logrado denotar un objeto, y podremos reconocerlo por sus propiedades, si bien del mero hecho de que hayamos logrado referirnos con esa expresión no se siga la existencia de ese objeto. De todas formas se seguirá la existencia de un objeto, o sea que un predicado o su negación es veredadero por lo menos de un objeto. Si afirmamos en este contexto que algo existe, forzoso será que el universo de discurso de esta predicación no coincida con el de las predicaciones normales en este contexto real. Esto se debe al problema que ya vimos: si coincidiera, la palabra 'existe(n)' no serviría para nada, sería verdadera de todo y falsa de nada. Para darle sentido - el que comúnmente tiene- tenemos que am- 
pliar el universo de discurso admitiendo entes de los que sea falso decir que existen. O sea, inscribiendo 'existe(n)' al universo de discurso heterogéneo de entes reales e imaginarios. Estas predicaciones tendrán también su condición de identificación, y por tanto, necesitaremos de un nuevo sentido de 'existe(n)' que signifique 'hay en el universo heterogéneo un objeto que es la referencia de tal palabra o expresión'.

La verdad de un enunciado existencial de segundo orden (que asi distinguiremos los dos sentidos de 'existe(n)') será condición para que pueda darse una predicación de existencia de primer orden. Pero un enunciado existencial de segundo orden requerirá a su vez de un objeto al cual aplicarse, y el enunciado de que esta condición se cumple implicaría un enunciado existencial de tercer grado. Pero exponer así las cosas es peligroso. Según vamos escribiendo, los órdenes de predicaciones existenciales no dejarian de sucederse indefinidamente. Si nos preguntamos por los universos de discurso en cuestión, nos percatamos inmediatamente de que nos hallamos en las inmediaciones del sinsentido. El universo de discurso correspondiente a las predicaciones de segundo orden tendría que ser el de los objetos imaginarios, reales o que no son ninguna de las dos cosas. Aquí podríamos incluir a los entes contradictorios. Pero en el siguiente paso ya no sabríamos qué hacer. Es conveniente, por tanto, desechar esta terminología engañosa. Para esto bastará con ver las cosas directamente, sin la ayuda de esquemas previos. Preguntémonos con respecto a los existenciales de primer orden, qué condición es necesaria, con relación al sujeto, para lograr una predicación. La respuesta es la de Frege y Russell: es necesario que la frase-sujeto nos proporcione un conjunto de propiedades, un concepto, en el lenguaje de Frege, una función proposicional en el de Russell. No es difícil ver que el universo de discurso cuyos miembros son los conceptos, o- las funciones proposicionales, es el mismo a que nos hemos referido con tan engañosa expresión 'universo heterogéneo de entes reales e imaginarios'. Porque, ¿cuál es nuestro criterio para aceptar un candidato en este universo de discurso? Es el mismo que tenemos para aceptar algo como un concepto. Si no logramos expresar un conjunto de propiedades, no podremos predicar existencia, no tendrá sentido, y por tanto, no habremos invocado un objeto de ese universo heterogéneo. Dije que no tendría sentido y la explicación es obvia: si no se da un conjunto de propiedades, sino solamente un objeto, no podríamos concebir lo que sucedería si la proposición existencial fuera falsa, o lo que es lo mismo, nada diríamos, la oración no expresaría un enunciado.

Cuando decimos que un concepto existe, tampoco podemos identificar lo mismo que cuando decimos que el concepto es inútil, que se aplica a muchos objetos, o que es obscuro. Decir que un concepto existe es decir que cierta expresión tiene sentido, que pertenece a un lenguaje en el que se le han dado las convenciones necesarias. Obsérvese que el sentido que se ha 
dado aquí a 'expresión' es el de fonema o garabato, y que, cuando una expresión, en este sentido, es el sujeto lógico de una oración, es ella misma la que aparece como sujeto de la oración, y no es nombrada, con lo que la identificación está asegurada. Claro está que es posible hablar de la existencia de un fonema, como objeto físico, pero hemos roto con esta explicación la cadena que parecía interminable de universos de discursos de órdenes sucesivos.

\section{Los adjetivos cuantificadores}

El hecho que voy a considerar fue primero presentado por Moore. Strawson se basa en él para dar un criterio de cuándo un sujeto y predicado gramaticales son, respectivamente, sujeto y predicado lógicos. Consideremos las siguientes oraciones.

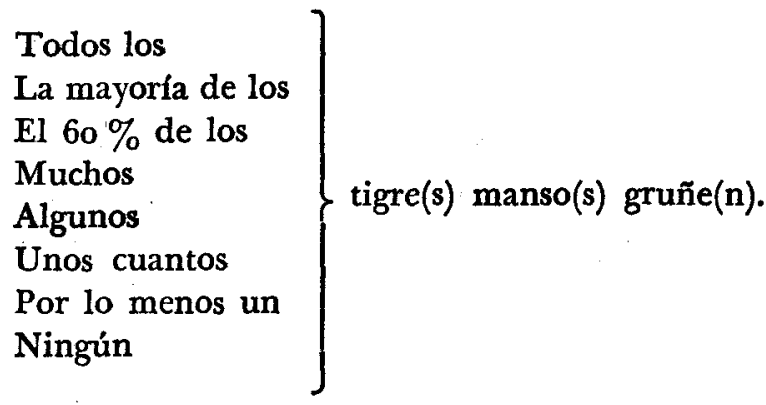

En lo que difieren estas oraciones, irrelevancias aparte, es en el adjetivo cuantificador con el que comienzan. Todas ellas son perfectamente significativas. Lo mismo sucede cuando sustituimos 'gruñe(n)' por 'no gruñe(n)'. Nos importa aquí que puedan ser significativas; rarezas como la de la oración "todos no vuelan" (a comparación de "ninguno vuela" o mejor, "no vuela ninguno") pertenecen a otro género de discusión, el caso es que tengan sentido. Esto mismo sucede con todos los predicados normales (éste es el criterio de Strawson): si tienen sentido con un cuantificador, lo tienen con todos los demás. Así se comportan todos los predicados que categorialmente competen a los tigres: que son hermosos, que tienen elástica la piel, que tienen amibas. $Y$, en general, cuando un predicado no tiene sentido de los tigres bajo un cuantificador, tampoco lo tiene bajo ningún otro.

En estos casos el predicado gramatical 'existe(n)' se comporta anormalmente: si sustituimos 'gruñe(n)' por 'existe(n)', en las oraciones anteriores, encontramos que las tres primeras no tienen sentido. Pero el predicado 'no existe(n)' se comporta en forma aún más extraña: no parece tener sentido con ninguno de los cuantificadores. Aquó debe advertirse una peculiaridad 
del castellano: si se invierte el orden de las palabras en estas oraciones, hay algunas que recobran el sentido, por ejemplo "no existen muchos tigres" o "no existe ningún tigre". Pero estos casos pueden ser interpretados en la misma forma en que serian los demás, bajo la idea de que la existencia no es un predicado.

Sinsentidos semejantes pueden encontrarse con la palabra 'hay' que puede tomarse sin peligro en este contexto como sinónimo de 'existe(n)', aunque para otros efectos pudieran tener distintas implicaciones. Tampoco, y en el mismo sentido, 'hay' es un predicador ordinario. "Hay todas las elefantas", "Hay la mayoría de las elefantas" "Hay el 70\% de las elefantas" no parecen tener sentido.

Para explicar estos hechos recurrimos a la idea de que la existencia no se predica de los miembros de la clase de los tigres como se predica que gruñan. En todas las oraciones que hemos considerado, la expresión que sirve de sujeto nos indica de qué tantos tigres mansos predicamos que gruñan. Algunos de los adjetivos cuantificadores cumplen esta función indicando de qué proporción de los tigres mansos se dice que gruñen. Tales son 'Todos', 'La mayoría', 'Más de la mitad', 'Una tercera parte', en general cualquiera en que se establezca un porcentaje. Hay cuantificadores que hacen esto en forma exacta, otros fijan la proporción con alguna ambigüedad $v$. gr., 'la mayoria' - 'más de la mitad'. Hay otros cuantificadores que no nos dicen qué proporción de tigres mansos gruñen, sino qué número. Tales son 'al menos un', 'dos', 'tres', 'algunos', 'unos pocos', 'muchos', etc. Entre estos cuantificadores también tenemos que unos lo hacen en forma exacta y otros dejan cierta vaguedad. Estos últimos no sólo son vagos porque nunca fijen un número con precisión, sino que además el vago número que determinen en cada caso depende del contexto de comparación en que sean usados.

Los adjetivos del primer género, puesto que establecen una parte proporcional de una clase, dan lugar a oraciones sin referencia cuando la clase sobre la que se establece la proporción es vacía. Los del segundo género también dan lugar a una falla de referencia cuando no hay miembros de la clase de la que toman un número. Cuando nos encontramos en un contexto de realidad y los predicados de las oraciones se predican de grupos, más o menos extensos y determinados, de objetos reales, decir que una clase es vacía es indicar que de ningún objeto real puede decirse que pertenece a esa clase. Pero, cuando en este contexto de realidad aparece el predicado 'existe(n)' no puede ser el caso que la predicación sea acerca de los grupos de objetos que constituyen el sujeto lógico de los demás predicados. El hecho de que la clase presupuesta sea vacia, no hace que la predicación de existencia sea nula, sino que la hace falsa. Cuando el predicado es la existencia, no puede presuponerse que la clase sujeto no es vacía, porque es eso lo que se afirma, no puede ser ella el sujeto lógico del enunciado. Pero si los adjetivos cuan- 
tificadores de proporción no tienen sentido, si no hay de dónde establecer la proporción, si presuponen que la clase no es vacía entonces no pueden usarse para hacer enunciados existenciales sin que la condición presupuesta coincida con lo que quiera afirmarse. El resultado es, pues, el siguiente: si el adjetivo establece el $100 \%$ (todos), entonces, o se produce una tautología referencial, o nos quedamos sin entender nada. La tautología referencial sería como esto: si presuponémos que la clase no es vacía, es porque encontramos que hay objetos que pertenecen a ella, pero, si en este contexto de realidad, sólo objetos reales pueden pertenecer a ella, entonces, todos los miembros de la clase existen. Debe observarse, sin embargo, que aquí se ha tenido que recurrir a un uso de 'existe(n)' - su aplicación a cosas_, que no puede ser informativo, pues no hace otra cosa que repetir que la función referencial de la frase-sujeto fue cumplida. Cuando decimos que "todos los tigres mansos existen" no tiene sentido, no queremos indicar que no pueda dársele ninguno, sino que si se le da, el resultado será una tautología referencial, es decir, una oración que sólo afirma su propia capacidad de referir, pero que no predica nada.

Con los demás adjetivos proporcionales sucede, además, que su uso ordinario, salvo que se indique lo contrario, no sólo implica que la clase sujeto no es vacía, sino que además la proporción que se indica, y sólo esa proporción, tiene el atributo predicado. Asl, cuando digo que el 7o \% de los tigres mansos existen implico que hay un $30 \%$ que no existen, lo cual es una contradicción. Por esta razón, las oraciones existenciales que estén cuantificadas con adjetivos proporcionales distintos de '100\%', (y obviamente del 'o \%') se acercan más al sinsentido.

Con los adjetivos cuantificadores que indican una cantidad (vaga o exacta), pueden hacerse enunciados existenciales perfectamente significativos. Pero en estos casos es claro - y esto es lo que queremos decir cuando decimos que la existencia no es un predicado lógico- que no se presupone que la clase sujeto no es vacía. Es eso precisamente lo que se afirma. Se dice que la clase no es vacía. El adjetivo cuantificador no dice cuántos miembros de una clase presupuesta tienen el predicado, sino cuántos miembros tiene la clase, cuán extensa es. Creo que ha quedado claro que estos adjetivos cumplen aquí un papel distinto y que pueden hacerlo debido a que son capaces de fijar (con la vaguedad o precisión que les compete a cada uno) una cantidad — sin necesidad de referirse a una clase, puesto que no fijan una proporción. No es nada misterioso, por otra parte, que las existenciales negativas no tengan sentido tampoco con estos cuantificadores. Para comprenderlo distingámoslas primero de las negaciones de las que sí tienen sentido. No es lo mismo decir "es falso que haya tres tigres mansos" (hay más, hay menos, no hay ninguno) que decir "tres tigres mansos no existen". Esta última oración no puede tener sujeto, pues ¿cuáles pueden ser esos tres tigres, dentro de la clase de los ti- 
gres, que carecen de existencia? Podemos decir cuál es la extensión de una clase, cuántos miembros tiene, podemos aun indicar cuál es la exclusión de una clase, o sea, cuántos objetos que pertenecen a otras clases no pertenecen a ésta. Pero decir que "tres tigres mansos no existen" no es ninguna de estas cosas. $Y$ el mayor sentido que podemos darle es el de una contradicción referencial. Nos referimos a tres tigres, y luego afirmamos su no existencia, es decir, que hemos fracasado en la referencia.

\section{El contraejemplo de Strawson}

En un artículo reciente Strawson ${ }^{1}$ presenta un tipo de contextos en los que a su entender la existencia cumple el papel de predicado. Como ya indiqué, la tesis corriente (Frege) es la que sostiene que en las oraciones existenciales el sujeto lógico no es el mismo que en las demás, sino el concepto que en las oraciones comunes constituye la connotación del sujeto. Se piensa que lo afirmado de ese sujeto, del concepto, es que se aplica con verdad por lo menos a un objeto. La tesis de Strawson es que este modelo de interpretación de las oraciones existenciales no es siempre conveniente, y propone otro modelo en el que, según dice, se preserva "un papel predicativo a 'existe' sin dejar caer a la expresión-sujeto en el un tanto ingrato papel de término singular que se refiere a un concepto".

La forma en que se intenta mostrar esto es la que sigue. Se muestran las rarezas que el predicado gramatical 'existe(n)' presenta frente a los adjetivos cuantificadores, hecho que se explica mediante la idea de clase presupuesta que hemos utilizado, pero sin mencionar una formulación como la nuestra de la tesis que niega que la existencia sea un predicado. De allí se desprende un criterio (test) para decidir si un predicado gramatical es también un predicado lógico. "Dados un sujeto y un predicado gramaticales, es condición necesaria para que cuenten como sujeto y predicado lógicos respectivamente, el que, si el sujeto gramatical puede ser precedido de cualquier adjetivo cuantificador, admita entonces la precedencia de todos los demás." De acuerdo con este criterio la existencia no es un predicado lógico porque, como hemos visto, admite algunos de los cuantificadores, pero no todos.

Pero hay contextos en los que 'existe(n)' admite todos los cuantificadores. Supongamos que llegamos a un lugar donde se están haciendo actos de predicación acerca de diversos individuos. Si preguntamos quiénes son esos personajes, se nos puede explicar: "la mayoría son imaginarios, pero una minoría existe", o "todos ellos existen".

Lo mismo sucede con los personajes que aparecen en un diccionario clásico. Podemos explicar "más de la mitad de ellos existieron, pero una buena parte son fruto de la imaginación de los antiguos".

1 "Is Existence Never a Predicate?", Critica No 1, vol. I, Enero de 1967. 
En estos contextos, el predicado gramatical 'existe(n)' cumple con el criterio expuesto renglones arriba. Entonces se pregunta Strawson si convenga decir que la condición es necesaria pero no suficiente y seguir manteniendo que la existencia tampoco es en estos contextos un predicado, o declarar que aquí la existencia sí es un predicado y explicar este hecho con un modelo distinto. $Y$ puesto que nada le obliga a tomar el primer camino, Strawson intenta el segundo. Supongamos que alguien objetara - sigo su discursoque si en estas oraciones la existencia es afirmada, no puede ser presupuesta y que es ese el fundamento del criterio que hemos dado; la respuesta de Strawson sería que hay aqui una confusión. "Podemos seguir manteniendo el principio de que donde se usan los adjetivos cuantificadores en la forma en que 'la mayoria' se usa siempre, y no como 'algunos', la clase-sujeto es una clase presupuesta. Sólo que tenemos que detenernos a considerar cuál es esa clase." La respuesta que propone es la siguiente: se trata de una clase heterogénea que se divide en dos clases fundamentales, la de los personajes existentes y la de los míticos. En este modelo 'existe(n)' se presenta como un verdadero predicado, y no de conceptos, sino de miembros de la clase heterogénea. En los enunciados existenciales generales se presupone que la clase heterogénea no es vacía, cuando el sujeto es 'personajes', y en las existenciales particulares, se presupone (es precondición para lograr un enunciado) que el sujeto identifica no a un personaje histórico, sino a un personaje histórico-o-legendario.

Después, Strawson dice que no debemos oponer este modelo al de los conceptos en todos los casos, porque serfa tomar demasiado en serio nuestros modelos, con lo que desatenderíamos "la grata fluidez de nuestro pensamiento".

Mi opinión es la de que no es cierto que se trate de dos modelos. Creo que se trata del mismo, y que el lenguaje de la clase heterogénea es menos claro que el de conceptos. Es admisible la traducción de proposiciones como "la mayoría de los personajes de los que hablamos existen", o "la mayor parte de los héroes que aparecen en este diccionario existieron", por proposiciones como las que siguen "la mayoría de los nombres que estábamos usando se refieren a individuos reales" o "la mayoria de las connotaciones de los nombres que usábamos se aplican con verdad a un solo individuo". También pudo decirse "la mayoría de las predicaciones que hicimos pertenecían a un contexto de realidad, pero una minoría se referían a mitos e invenciones". No hay duda de que por lo menos las proposiciones expresadas en el primer modo (personajes de los que hablamos) siempre tienen un equivalente expresable en el segundo modo (conceptos). Equivalentes en el sentido de que no pueden tener distinto valor de verdad. Se puede discutir ahora si expresan, además, la misma proposición. Una razón para negarlo serfa decir que unas se refieren a personajes y otras a conceptos. Se diría que 
tienen distinto tipo de sujeto lógico. Pero, ¿̨en qué nos basamos para probar esto último? Solamente en que los sujetos gramaticales de los dos tipos de oración son distintos. En un caso aparece la palabra 'personajes', en otro, la palabra 'conceptos', o 'nombres'. Si se nos pregunta acerca de qué hablan las distintas oraciones, diremos que unas hablan de personajes y otras de conceptos. Pero es un error fiarnos de la expresión 'hablar acerca de'. Esta expresión no significa lo mismo que 'sujeto lógico'. Si decimos "las elefantas cretinas no existen", y se nos pregunta de qué hablamos, diremos que de las elefantas cretinas. Cuando decimos que hablamos acerca de algo, lo único que hacemos es repetir el sujeto gramatical de nuestras oraciones. La expresión 'hablar de' no pertenece al metavocabulario del lógico, y su función se reduce a lo que dije antes: indicar el sujeto gramatical. Se trata de una expresión anterior a la distinción sujeto lógico/sujeto gramatical. Cuando nós preguntamos cuál es el sujeto lógico, lo que hacemos es preguntar por condiciones de identificación, y esto es ya más que preguntar acerca de qué hablamos.

Examinemos el esquema de Strawson más de cerca. ¿Cuál es el universo de discurso de predicaciones como "la mayoría de los personajes existen"? -Es el universo de discurso heterogéneo de los personajes existentes y no existentes. La clase sujeto, la que se presupone (como no vacía) es un subconjunto de este universo: los personajes mentados en la conversación; de ellos se habla. Si nos preguntamos ahora cuál es el tipo de existencia presupuesta por las predicaciones relativas a este universo de discurso, comprobaremos que no es la misma que se presupone cuando se dice "Napoleón venció en Austerliz" y que es la misma que tiene la predicación "Napoleón existio", o sea la existencia de un concepto. Es necesario que 'Napoleón' tenga connotación para poder afirmar que existe. Eso es lo que se necesita para que algo pertenezca al universo de discurso de los personajes: que tenga connotación, 'que sea un concepto. $\mathrm{Y}$ así como podemos decir "el concepto $C$ es satisfecho por un objeto de este otro universo de discurso" o "el concepto $C$ acaba de ser usado", también podemos decir "la mayoría de los conceptos que han aparecido como sujetos en estas oraciones son satisfechos al menos por un individuo del universo de discurso de los entes reales".

Tanto en "Napoleón venció en Jena" cuanto en "la mayoría de los personajes mentados existen" hablo de personajes. Esto, abusando de la pobreza lógica de la expresión 'hablo de' y de la palabra 'personajes'. La diferencia capital entre las dos predicaciones -que encierra el contenido de la tesis que niega a la existencia la calidad de predicado lógico- es que para que la primera predicación se logre es necesario que se identifique a Napoleón en la historia, y para que la segunda se logre es necesario tener palabras connotativas como sujeto, aunque no identifiquen a nadie en la historia, ninguna de ellas, en cuyo caso la predicación se lograría y sería falsa. 
Es por eso inconveniente dar un criterio como el de Strawson. Los hechos relativos a los adjetivos cuantificadores deben entenderse siempre en relación a un contexto de identificación particular. Cuando pensamos que hay casos que no tienen sentido es porque permanecemos en el mismo contexto de identificación; lo que hacen los ejemplos de Strawson es cambiar el contexto y a eso se debe que las proposiciones en cuestión tengan sentido. Podemos dar un sentido a "la mayoría de los tigres existen", mientras contemos en nuestro universo de discurso a tigres imaginarios y reales.

\section{Las tautologias referenciales}

D. F. Pears expone la noción de 'tautología referencial' en un artículo llamado Is Existence a Predicate? con las siguientes palabras: " "si la frase-sujeto de un enunciado singular implica referencialmente existencia, entonces, si el verbo afirma existencia, el enunciado será una tautología referencial, y, si el verbo niega existencia, el enunciado será una contradicción referencial, excepto bajo una de las siguientes tres condiciones: en primer lugar, que la implicación y la aserción sean acerca de mundos diferentes [real e imaginario]; en segundo, que se refieran a tiempos distintos; en tercer lugar, que sean acerca de distintos niveles [alucinaciones-cosas]". Si nos preguntásemos qué quiere decir que el sujeto implique referencialmente existencia, encontraremos la respuesta de Pears en el trozo que sigue: "... supongamos que hago un enunciado existencial singular 'esta habitación existe'. Hay algo obviamente raro en este enunciado. Porque la frase-sujeto 'esta habitación' implica que esta habitación existe por hacer referencia a ella, y el verbo 'existe' no hace sino volver a afirmar esa existencia".

Es preciso aclarar aquí algunas cosas. Si la razón por la cual "esta habitación existe" es una tautología referencial fuera que la frase-sujeto implica referencialmente la existencia de esta habitación, y esto último se debiera a que la frase-sujeto hiciera referencia a ella, entonces "la Reina de Inglaterra existe" sería una tautología referencial. Otra cosa que conviene poner en claro, es que la referencia de 'esta habitación' no es el sujeto lógico de "esta habitación existe" como parece seguirse de los párrafos de Pears. Estos dos puntos son muy importantes. Su esclarecimiento nos apuntará una dificultad en la formulación de la tesis tal como la he presentado hasta ahora. Su exposición nos ayudará a dar con una interpretación adecuada de las tautologías referenciales.

Mi interpretación es la siguiente. En el enunciado "esta habitación existe" el sujeto lógico no es esta habitación. La razón se encuentra en una observación de Moore: "esta habitación pudo no haber existido" es una pro-

2 Aquinas Paper 38, Aquin Press, 1963. Reimpreso con leves alteraciones en Philosophical Logic, ed. P. F. Strawson, Oxford University Press, 1967 . 
posición verdadera. Si lo que afirma es que la existencia de esta habitación no es una verdad de la lógica, entonces se trata de una proposición necesaria. Ahora bien, el sujeto lógico de "esta habitación existe" y el de "esta habitación pudo no haber existido" parece ser el mismo. Supongamos que el sujeto lógico fuera esta habitación, en tal caso no podríamos predicar de ella su no existencia, porque una precondición para predicarla sería que la habitación existiera. Cuando decimos que esta habitación no existe por necesidad lógica, sino que su existencia es una contingencia que de hecho se ha dado, no es posible que lo que queramos decir es que la oración "esta habitación existe" pudo ser falsa. Lo que queremos decir es que otras oraciones que corresponden a este uso de la oración pudieron haber sido falsas. O dicho de otro modo: este uso particular de "esta habitación existe" pudo no haber sido empíricamente posible. Hay mundos posibles en los que ese uso, i. e., el que tiene la misma referencia, no puede darse: porque no hay esa habitación. ¿Cuál es pues el criterio de identificación de nuestro enunciado? En un mundo posible sin esta habitación, lo que decimos ahora con la oración "esta habitación existe" (si bien no lo hubiéramos podido decir con esta oración) sería falso. La misma predicación (con otra oración) se hubiera podido lograr sin que existiera este cuarto. Por lo tanto no es esta habitación el sujeto lógico. No podría, en cambio, predicarse lo mismo si no supiéramos de algunas propiedades de esta habitación. Un conjunto más o menos vago de propiedades es el sujeto lógico de la oración.

Cuando se formula la tesis diciendo que en las oraciones existenciales el sujeto lógico es la connotación ordinaria de la frase-sujeto, se está desatendiendo esta provincia gramatical de los demostrativos, si bien se trata de casos extraños y rara vez útiles. De esta formulación, y puesto que los demostrativos no connotan, Russell concluye que "esto existe" es un sinsentido. Pero no es así, para que pueda darse una predicación existencial es necesario que sea aislado un conjunto de propiedades (se identifique una función proposicional), pero no es el único modu de hacerlo el de proporcionar una frase connotativa. Se puede identificar ese conjunto de propiedades a través de un objeto real, si bien esta forma de hacerlo garantiza que la predicación de existencia sea verdadera. A esto se debe que esas oraciones no puedan dar lugar sino a enunciados verdaderos, a que una condición para que mediante esa oración se identifique el sujeto lógico sea la verdad del enunciado. Subrayé 'mediante esa oración' porque es esto precisamente lo que hace que la cosa no sea el sujeto lógico. Es también necesario identificar a esta habitación para lograr el enunciado, pero ésta es una contingencia de la oración y no de la predicación en cuestión. Hay formas de hacer el mismo enunciado sin que para identificar al sujeto lógico sea indispensable la verdad de la proposición. Esto mismo explica la falacia de Moore que corre 
asi: "esto podría no haber existido" es verdadera, puesto que de lo contrario esto existiría por necesidad lógica; de lo que se sigue que "esto existe" tiene sentido; se concluye que la existencia sí puede predicarse de las cosas sin absurdo lógico.

Hugo MaRgáin

INSTTtuto DE INVEstigaciones Filosóficas

Universidad Nacional Autónoma de México 